\title{
Targeted gluteal exercise versus sham exercise on self-reported physical function for people with hip osteoarthritis (the GHOst trial - Gluteal exercise for Hip Osteoarthritis): a protocol for a randomised clinical trial
}

\author{
Adam Ivan Semciw ${ }^{1,2^{*}} \mathbb{D}$, Tania Pizzari ${ }^{1}$, Stephanie Woodley ${ }^{3}$, Anita Zacharias $^{4}$, Michael Kingsley ${ }^{5}$ \\ and Rod A. Green ${ }^{4}$
}

\begin{abstract}
Background: Clinical practice guidelines recommend exercise as the first line of management for hip osteoarthritis, yet high-quality evidence from Cochrane reviews suggest only slight benefits for pain and physical function; and no benefit on quality of life (low-quality evidence). However, the scope of physical impairments identified in people with hip osteoarthritis may not have been adequately addressed with targeted rehabilitation options in previous randomised controlled trials (RCTs). Potential targeted options include gait retraining to address spatio-temporal impairments in walking; motor control training to address deep gluteal (gluteus minimus) dysfunction; and progressive, high-intensity resistance exercises to address atrophy of the gluteal muscles. The aim of this study is to investigate the effect of a targeted gluteal rehabilitation programme that incorporates gait retraining, motor control and progressive, high-intensity resistance-strength training, to address physical activity levels and self-reported physical function in people with mild to moderate disability from hip osteoarthritis.

Methods: Ninety people diagnosed with mild to moderately disabling hip osteoarthritis will be recruited and randomised to receive one of two exercise programmes (sham or GHOst programme). Interventions will be 12 weeks in duration, with weekly, supervised physiotherapy sessions, and daily home exercises. Both groups will receive standardised education. Outcomes will be assessed at baseline, 7 weeks, 13 weeks (primary time-point) and 25 weeks. The primary outcome will be self-reported physical function measured with the Western Ontario and McMaster Universities Osteoarthritis Index (WOMAC). Secondary outcomes include physical activity measured with a tri-axial accelerometer, physical function tests, self-reported physical activity, isometric hip-muscle strength tests, hip-related patient-reported outcome measures, pain thoughts and depressive symptoms, quality of life, global rating of change, gluteal-muscle activity (electromyography (EMG)) and gluteal-muscle size and adiposity (magnetic resonance imaging (MRI)).

(Continued on next page)
\end{abstract}

\footnotetext{
* Correspondence: a.semciw@latrobe.edu.au

'Department of Rehabilitation, Nutrition and Sport; La Trobe University,

Bundoora, VIC, Australia

${ }^{2}$ School of Health and Rehabilitation Sciences, The University of Queensland,

St Lucia, QLD, Australia

Full list of author information is available at the end of the article
}

(c) The Author(s). 2018 Open Access This article is distributed under the terms of the Creative Commons Attribution 4.0 International License (http://creativecommons.org/licenses/by/4.0/), which permits unrestricted use, distribution, and reproduction in any medium, provided you give appropriate credit to the original author(s) and the source, provide a link to the Creative Commons license, and indicate if changes were made. The Creative Commons Public Domain Dedication waiver (http://creativecommons.org/publicdomain/zero/1.0/) applies to the data made available in this article, unless otherwise stated. 
(Continued from previous page)

Discussion: This will be the first study to compare a targeted gluteal rehabilitation programme to a sham exercise programme. The targeted GHOst programme includes exercises designed to address gait impairments as well as glutealmuscle atrophy and dysfunction.

Trial registration: Australian New Zealand Clinical Trials Registry, ID: ACTRN12617000970347. Registered prospectively on 5 July 2017. Protocol version 3.0.

Keywords: Hip osteoarthritis, Exercise, Buttocks, Gluteal, Gait, Clinical trial

\section{Background}

Osteoarthritis is a chronic, debilitating condition affecting many people worldwide. In 2016, approximately $4 \%$ of the global population had a diagnosis of osteoarthritis, with the prevalence reaching $9 \%$ in countries such as the United States, Germany and Canada [1]. Osteoarthritis typically presents in the weight-bearing joints of the lower limb such the hip and knee [2]. Symptoms in people with hip osteoarthritis usually deteriorate slowly [3], and this likely contributes to a reduction in physical function [4,5] when undertaking simple activities such as walking. The symptoms and reduction in physical activity are thought to contribute to elevated Body Mass Index (BMI), weakness, stiffness, psychological distress, poorer quality of life [6], and greater personal and societal burden $[7,8]$.

Clinical practice guidelines recommend exercise and education as first-line management for osteoarthritis of the hip or knee [9-13]. The ultimate goal of exercise participation in people with osteoarthritis is to improve symptoms, physical function and quality of life. Theoretically, the improvements obtained with exercise are achieved by addressing bio-physiological (e.g. muscle strength, joint stability, cartilage integrity) and psychosocial impairments present in this patient group $[4,14-18]$. A Cochrane review and meta-analysis concluded that there is moderate- to high-quality evidence that exercise has moderate benefits for pain and physical function, and slight benefits for quality of life in people with knee osteoarthritis [19]. However, exercise interventions for those with hip osteoarthritis appear to be less efficacious. High-quality evidence from a Cochrane review and meta-analysis suggests that exercise has only slight benefits for pain and physical function in people with hip osteoarthritis [20]; and no improvement in quality of life (low-quality evidence) [20]. A more recent, high-quality randomised controlled trial (RCT) supplements these findings to suggest that exercise combined with manual therapy is no better than a sham intervention (turned-off ultrasound) for improving pain and physical function in this patient group [21]. It is possible that the principles of exercise training programmes (frequency, intensity, type, duration and progression) have not been optimised to target the specific impairments associated with hip osteoarthritis, and these training principles are an important consideration for clinical prescription and future research [20, 22, 23].

Targeting specific impairments might be important to optimise outcomes of rehabilitation for people with hip osteoarthritis. Peak hip-abductor isometric strength, and hip-adduction moment during gait (a surrogate measure of hip-abductor strength) are significantly associated with pain while walking in people with hip osteoarthritis [24]. Furthermore, hip-abductor muscle atrophy is present in those with hip osteoarthritis, and this is associated with radiological and clinical severity $[25,26]$. The use of progressive, high-intensity resistance-strength training for the hip abductors may potentially have a significant impact on symptoms during functional tasks like walking, and ultimately affect physical function and quality of life. There are, however, no studies that have investigated high-intensity resistance-strength training in this population [14, 20, 21]. In addition, individuals with symptomatic hip pathology present with biomechanical [27] and spatio-temporal [28] gait impairments that could potentially be addressed with rehabilitation. For example, reduced peak hip extension during gait is evident in early hip pathology [29] as well as established hip osteoarthritis [27, 30]. It has been theorised [31, 32] that loss of terminal hip extension reduces the stimulus of the deep anterior hip muscles (e.g. gluteus minimus (GMin)) to contract [33]. Weak or inefficient anterior hip muscles may consequently leave the anterior-superior hip joint vulnerable to further shearing, injury and degeneration [34, 35]. Indeed, GMin atrophy is present during end-stage hip osteoarthritis [26, 36], with a particular susceptibility of anterior GMin [37], and persists following hip surgery $[38,39]$. It is, therefore, important to consider gait retraining and motor control strategies to correct for these biomechanical and muscle impairments.

Gait retraining has been recommended in recent clinical guidelines for the management of hip osteoarthritis [11]; however, this is based on weak evidence including expert opinion. There are currently no studies that include gait retraining during rehabilitation in people with hip osteoarthritis. Clearly, the scope of rehabilitation options for targeting specific impairments in people with hip osteoarthritis has not been fully explored. Potential options include, high-intensity resistance-strength training, gait retraining and motor control strategies targeting 
muscle segments (e.g. GMin anterior) that are known to atrophy in this population [37].

Using a parallel RCT design, the aim of this study is to compare a targeted gluteal rehabilitation programme that incorporates gait retraining, motor control and progressive, high-intensity resistance-strength training, to a sham exercise intervention for addressing self-reported physical function in people with mild to moderately disabling hip osteoarthritis.

\section{Methods}

This study will be reported according to the Consolidated Standards of Reporting Trials (CONSORT) 2010 guidelines [40] and the protocol developed according to Standard Protocol Items: Recommendation for Interventional Trials (SPIRIT) (Additional file 1) [41, 42]. The study design and protocol follows the OARSI clinical trials recommendations for the conduct of clinical trials for hip osteoarthritis as set out in Lane et al. [43]. This study will compare the effect of a 12-week, targeted, physiotherapy-supervised gluteal exercise programme, with a 12-week physiotherapy-supervised, sham exercise programme in people with hip osteoarthritis. The trial has been registered on the Australian New Zealand Clinical Trials Registry (ACTRN12617000970347) with a Universal Trial Number (UTN) U1111-1192-1408. Where appropriate, approval for trial modifications will be sought by the relevant ethics committee and updated on the ANZCTR. The study will take place in three Australian cities (Bendigo, Melbourne, Brisbane) and one New Zealand city (Dunedin) involving physiotherapy clinics across the public and private sectors.

\section{Ethical approval and consent}

This trial adheres to the principles of the Declaration of Helsinki, and has received ethical approval from the Bendigo Health Care Group Human Research Ethics Committee (HREC/17/BHCG/3); La Trobe University HREC; The University of Queensland (20,170,001,541/ HREC/17/BHCG/3) and Otago University HDEC (17/ $\mathrm{STH} / 205)$. All participants will provide informed, written consent before commencing the study.

\section{Design}

This is a two-arm, assessor- and participant-blind, parallel, randomised clinical trial, with a 12-week intervention period and outcomes measured at baseline, 7 weeks, 13 weeks and 25 weeks (Fig. 1).

\section{Participants}

\section{Recruitment}

Potential participants with hip osteoarthritis will be recruited via local print media and through advertising flyers distributed through participating health providers and community notice boards. Further advertising through social media and radio and online advertising service will be conducted. Interested volunteers will contact chief investigators (RG, AS, TP, SW) at one of the four participating sites via telephone or email and will be screened (by telephone) for eligibility.

\section{Eligibility}

The inclusion criteria are as follows: mild to moderate disability from idiopathic (primary) hip osteoarthritis in accordance with the American College of Rheumatology [44] as defined by:

(i) Aged $>35$ years

(ii) Pain in the hip or groin for more than 3 months

(iii) Pain intensity over the past week of $\geq 30$ or higher on a $100-\mathrm{mm}$ visual analogue scale (VAS) during functional tasks like walking, climbing stairs or climbing in/out of a car

(iv) Radiographic confirmation of hip osteoarthritis with a Kellgren-Lawrence score $\geq 2$ [45]

(v) Mild to moderate disability indicated by:

a. Oxford Hip Score of 25-45 indicating mild to moderate disability $[46,47]$

b. Still able to reciprocally ascend and descend 10 stairs unaided [48]

c. Still able to safely walk one city block, and

d. Able to jog $5 \mathrm{~m}$

(vi) Satisfactory completion of an adult pre-exercise screening tool (https://www.essa.org.au/wp-content/ uploads/2011/09/Screen-tool-version-v1.1.pdf)

Exclusion:

(i) Other musculoskeletal lower limb or back conditions requiring assessment or treatment in the last 6 months

(ii) Primary complaint of gluteal tendinopathy (clinical diagnosis), low back pain or referred back pain (Additional files 2 and 3)

(iii) History of hip trauma or surgery on either the affected or unaffected side

(iv) Known knee joint pathology that may impact on the ability to perform the intervention or reduced knee range of motion $\left(<90^{\circ}\right.$ flexion)

(v) Corticosteroid use (oral or intra-articular) in the past 3 months

(vi) Neurological impairment or condition affecting lower-limb function

(vii) Conditions or factors affecting ability to take part in the exercise intervention, e.g. unavailable for a 12-week intervention period, routine use of gait aids, uncontrolled hypertension, or morbid obesity (Body Mass Index > 40) 


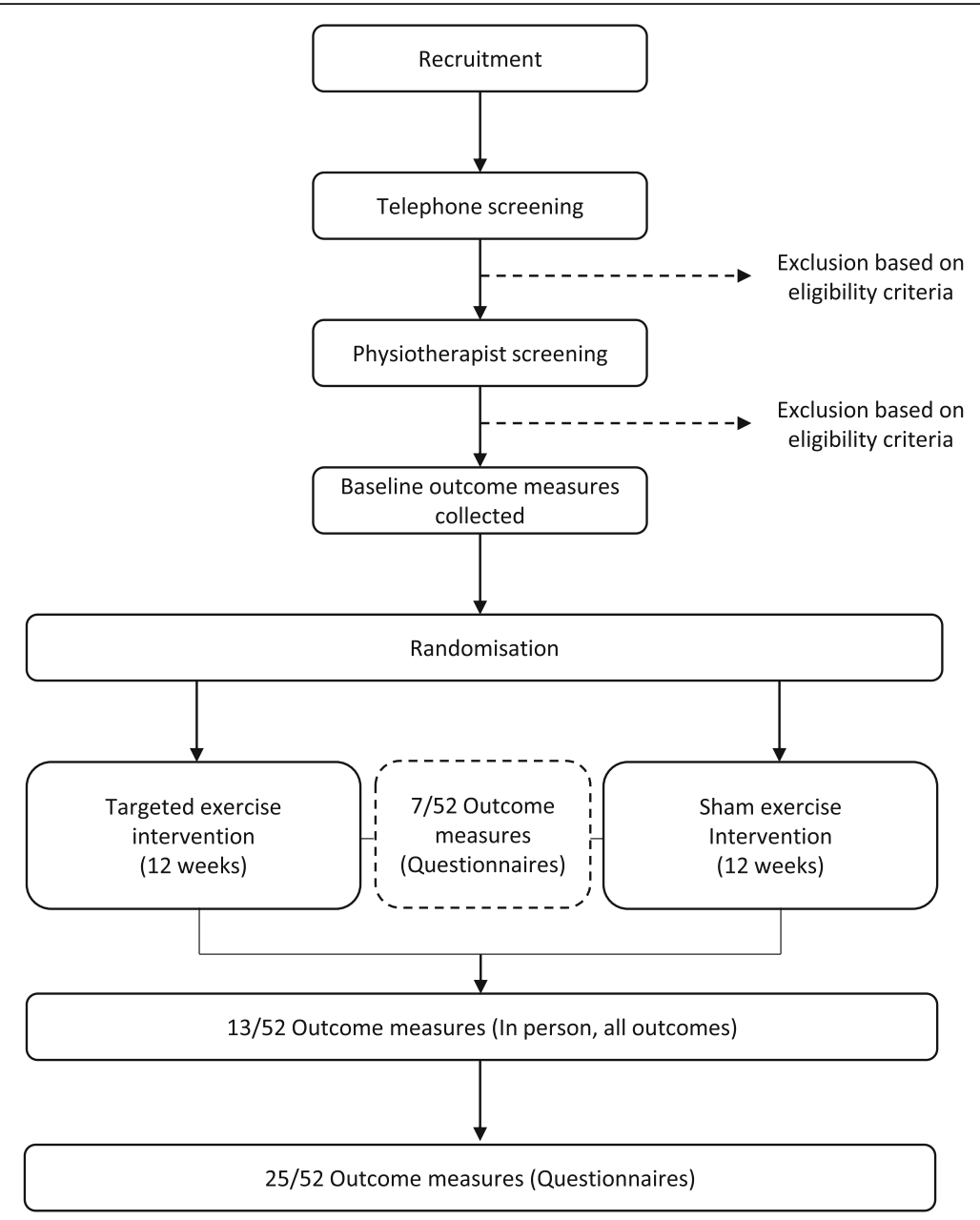

Fig. 1 Flow of participants through the study

(viii) Systemic inflammatory disease (e.g. rheumatoid arthritis)

(ix) Unable to write, read or comprehend English

In addition, participants at one site (Bendigo; total of 30 participants) will be asked to complete a magnetic resonance imaging (MRI) scan and undergo fine-wire electromyography (EMG) of the GMin and gluteus medius (GMed) muscles. These volunteers will be ineligible to participate if they have any contraindication to fine-wire EMG (e.g. fear of needles, taking blood-thinning medication) or MRI (e.g. pacemaker, claustrophobia).

\section{Study procedure}

\section{Telephone screening}

Interested volunteers will initially be screened over the telephone by one of the chief investigators at each of the four study sites. Telephone screening will aim to assess and exclude potential participants who do not fulfil the eligibility criteria. Reasons for exclusion at this stage include, but are not limited to; a clear diagnosis other than hip osteoarthritis; inability to complete a 12 -week intervention programme at a trial physiotherapy clinic; Oxford Hip Score outside the range of 25-45; unable to walk a city block, ascend/ descend a set of 10 stairs, or jog $5 \mathrm{~m}$; pain $<30 \mathrm{~mm}$ on a VAS during functional tasks; routine use of gait aids.

\section{Physiotherapy assessment}

Potential participants who satisfy the initial telephone screening will be invited to attend a physiotherapy screening examination. This screening session will include a physical examination to exclude gluteal tendinopathy and lumbar spine pain as the primary source of hip pain; and to exclude knee pathology that may affect their ability to complete the exercise programme. Screening for gluteal tendinopathy (clinical diagnosis) will be completed using the following protocol (Additional file 2): (1) tenderness to palpation over the greater trochanter (high sensitivity [85.7 (66.4-95.3)] suggesting that a negative test would rule out the condition [49]), in combination with (2) pain reproduction with the flexion, abduction, external rotation 
test (FABER) $[49,50]$ and (3) one other clinical test associated with gluteal tendinopathy (resisted external de-rotation test, resisted abduction test, single-leg stance test (30 s) [49, 51]. Lumbar spine referral as a primary source of pain will be screened by the following criteria (Additional file 3): (1) reproduction of hip symptoms with repeated lumbar flexion, lumber extension, or quadrant testing and (2) reproduction of hip symptoms with passive straight leg raise to $45^{\circ}$ hip flexion. The physiotherapist will also assess passive knee range of motion to ensure $>$ $90^{\circ}$ of pain-free movement in order to screen for serious knee pathology. If potential participants satisfy the remaining eligibility criteria, they will once again be instructed on the nature of the study, offered the opportunity to ask questions about the study, and invited to provide written, informed consent (Additional file 4).

\section{Randomisation, allocation and blinding}

A randomisation schedule will be generated by an investigator $(\mathrm{MK})$ who will have no contact with participants for the duration of the trial, including participant screening, baseline assessment, intervention, or other outcomes assessment. A web-based randomisation programme (https://www.randomizer.org/) will be used to generate the randomisation schedule, with a 1:1 allocation ratio, stratified by site. Group allocations will be concealed in serially numbered, opaque, sealed envelopes for each site. These envelopes will be posted to the trial physiotherapist at each site who will not be involved in participant screening or recruitment. The trial physiotherapist will open the envelopes sequentially according to participant number to determine the participant's group allocation prior to their first appointment (after eligibility screening, enrolment and baseline testing has been completed).

Assessor blinding All outcome measures will be assessed by a research assistant at each site who will be blind to participant group allocation. Participants will be instructed not to divulge any aspect of their intervention to the research assistant conducting follow-up assessments.

Participant blinding Participants will be blinded to group allocation (sham or Gluteal exercise for Hip Osteoarthritis (GHOst) protocol). They will be advised that they have an equal chance of being allocated to the either protocol. Participants will also be blind to the study hypothesis, so they are unaware which of the interventions is 'active'.

Physiotherapy blinding It is not possible to blind the trial physiotherapists to group allocation. Trial physiotherapists will, however, not be involved in assessment outcome measures.

\section{Interventions}

Participants will be randomly allocated to the GHOst intervention or the sham intervention. Both groups will receive standardised education for hip osteoarthritis delivered by a trial physiotherapist and supplemented with handouts. Education will focus on an understanding of the condition and general advice on physical activity. Participants will be asked to refrain from other active, supervised (e.g. from a health professional) non-pharmacological rehabilitation for their hip osteoarthritis for the duration of the trial.

Both groups (GHOst and sham) will receive once weekly, supervised physiotherapy sessions and a self-managed exercise programme over 12 weeks. The self-managed home exercise programme will consist of the same exercises prescribed during the supervised sessions. The initial physiotherapy session will be a one-to-one session for $1 \mathrm{~h}$, incorporating education and exercises, delivered by the trial physiotherapist. The additional supervised sessions will be 30 min and conducted in an individual or group setting (i.e. one to three participants per session), based on availability of participants and scheduling of the trial physiotherapist. Participants will only attend group sessions with other participants who have been randomised to the same intervention. Whether participants attend group or individual sessions will be documented by the trial physiotherapist, and accounted for as a potential covariate in statistical analysis. Daily exercise adherence (frequency and duration) will be monitored with an exercise diary [52].

\section{GHOst protocol: targeted gluteal intervention}

The GHOst protocol is composed of three phases. Gait retraining, motor control (anterior hip stability) and pelvic stability with global, high-intensity resistance-strengthen ing. Exercises within each phase are progressed based on quality (judged by the trial physiotherapist), symptoms (pain not exceeding $5 / 10$ on a numerical pain rating scale) and ability to complete the relevant dose.

Gait retraining The aim of the gait retraining component is to normalise, prevent or minimise gait-related impairments and symptoms that are commonly associated with hip osteoarthritis $[28,53]$. This includes pain with walking, reductions in stride length, decreased peak hip extension range of motion, decreased cadence, decreased gait speed, gait asymmetry and increased pelvic obliquity at push-off $[27,28,53]$. Techniques will be prescribed by the trial physiotherapist to normalise stride length and asymmetry. These include, but are not limited to, auditory cueing [54], backwards walking $[55,56]$ and instructions on techniques that may reduce pain when walking; for example, 'push more with your feet when you walk' $[57,58]$. 
Motor control (anterior hip stability) The second phase of the GHOst intervention aims to promote the function of the anterior hip muscles (particularly anterior GMin) using targeted, functional progressions of a split squat and bridge exercise. GMin appears to have a particular vulnerability to atrophy in people with hip pain $[26,32,37]$, especially the anterior portion [32, 37-39]. This phase will, therefore, have a focus on anterior GMin muscle recruitment using elastic exercise bands to promote active hip internal rotation [33, 59] in functional, closed-chain positions. Participants will be asked to complete up to five repetitions of 30-s holds for isometric exercises; three sets of 12 to 20 repetitions for dynamic exercises.

Pelvic stability with global strengthening The third phase of the GHOst intervention aims to improve pelvic stability and overall lower-limb and trunk strength and function. These exercises progress from isometric hip hitching [60-63], through to high-intensity resistancestrength training of multiple muscle groups (e.g. double-leg squat and dead-lift) with the aid of Theraband and power-bands (Aussie Strength, Smithfield, NSW Australia) to achieve the required intensity. The high-intensity resistance exercise component of this phase will use the Borg CR10 scale [64-67], asking participants to exercise at a 'heavy load' equivalent to Borg $\geq 5$ to $<7$. This intensity has been validated for use with hip exercises [66]. For the high-intensity resistance exercises, participants will be asked to perform three sets of 6 to 10 repetitions.

\section{Sham exercise protocol}

The sham protocol is similar to that being used in a $\mathrm{RCT}$ in people with greater trochanteric pain syndrome [61]. The sham programme differs to the GHOst proto$\mathrm{col}$ in that it is not aimed at strengthening the gluteal muscles, but includes generalised lower-limb, low-intensity resistance exercise. Participants are guided through sham gluteal, quadriceps' and calf exercises, predominantly in a seated position (unloaded).

\section{Physiotherapy treatment fidelity}

To maximise physiotherapy treatment integrity, all trial physiotherapists will have at least 2 years of musculoskeletal clinical physiotherapy experience, and will receive mandatory standardised training ( 1 day) before being assigned their first participant. Training will cover interventions to be implemented (sham and GHOst), the education to be delivered to participants and trial reporting (e.g. clinical note taking and adverse events). Training will incorporate techniques described by Main et al. [68] for training therapists to implement clinical trial interventions; including trainer-led teaching, role play, group discussions and ongoing clinical mentoring and support throughout the trial.

In addition to training, trial physiotherapists will receive a trial manual that includes an illustrative guide to exercise progressions. Trial physiotherapists will also document the exercises prescribed, adherence, use of pain medication or non-steroidal anti-inflammatory drugs (NSAIDS), and adverse events at each treatment session on standardised recording forms.

\section{Baseline assessments}

Demographic details including age, gender, height and weight will be recorded.

\section{Outcome measures}

The outcome measures are in accordance with those recommended by OARSI for clinical trials on people with hip osteoarthritis $[43,69]$.

\section{Primary outcome measure}

Self-reported physical function Self-reported physical function as assessed by the physical function subscale of the Western Ontario and McMaster Universities Osteoarthritis (WOMAC) Index Likert version 3.1 [70].

The physical function subscale of the WOMAC is a 17-item 5-point Likert scale ranging from 0 to 68 with higher scores indicating greater dysfunction. It is a valid and reliable self-administered tool [71] that is recommended for clinical trials in hip osteoarthritis [43], with a minimal clinically important difference of 6 units [72].

\section{Secondary outcome measures}

Physical activity accelerometry A tri-axial accelerometer (Link; Actigraph Corp., Pensacola, FL, USA) will be used as the objective, reliable measure of physical activity [73]. Participants will attach the accelerometer to a waistband anterior to the right hip for collection of movement data, at an acquisition frequency of $100 \mathrm{~Hz}$. Participants will remove the accelerometers when sleeping (to avoid sleep disturbance) and bathing (due to the lack of water-proofing) [74]. The accelerometer will be worn for a 7-day period in week 0 (prior to commencing the intervention) and week 12 (last week of the intervention).

Output data will be downloaded and analysed in 1-min epoch intervals using the manufacturer's software (Actilife ver.6; Actigraph Corp., Pensacola, FL, USA). The total time that the participant undertook sedentary, light, moderate and vigorous physical activity will be determined for each valid wear day using previously recognised algorithms [75]. The accumulated total of minutes of moderate and vigorous activity for the week will be 
summed to determine weekly total moderate to vigorous physical activity (MVPA, min/week) [76].

Physical function tests Recommended OARSI physical function tests for clinical trials on people with hip osteoarthritis [69] will be used, including the 40-m fast-paced walk test, stair-climb test and the chair-stand test. All three tests have acceptable reliability with small measurement error in people with hip osteoarthritis [77], facilitating the ability to measure changes over time.

Self-reported physical activity The International Physical Activity Questionnaire - short form is a self-administered questionnaire that aims to assess health-related physical activity [78]. Participants are asked to recall their physical activity over the last 7 days with regard to vigorous physical activity, moderate physical activity, walking and sitting.

Isometric hip-muscle strength Isometric hip strength will be assessed using handheld dynamometer $[79,80]$. Measurement of hip strength using hand-held dynamometry is reliable $\left(\mathrm{ICC}_{2,1}=0.76-0.95\right)[79,81]$. Hip abduction, adduction, flexion, extension, internal rotation and external rotation will be measured bilaterally (Table 1). The peak force $(\mathrm{N})$ produced from two trials separated by $15 \mathrm{~s}$ will be recorded for each action. Participants will be instructed to slowly build up their isometric strength against resistance until they reach their maximum, then maintain this level for $3 \mathrm{~s}$, and slowly relax. Consistent encouragement will be provided via standardised audio recordings. The order of the action tested (e.g. flexion) will be randomly assigned between participants using marked cards that are selected out of a hat, and will remain consistent from pre-test to post-test. Torque $(\mathrm{Nm})$ will be determined $(T=$ Force (N) $\mathrm{x}$ moment arm (m)) and normalised to body mass $(\mathrm{Kg})$ with the final unit of measurement being $\mathrm{Nm} / \mathrm{Kg}$.

Pain The average pain over the previous week will be assessed using an 11-point numerical rating scale (NRS), anchored by $0=$ 'no pain' and $10=$ 'worst pain possible'. The minimal important clinical difference is 1.8 [82].

Global hip questionnaires Hip disability and Osteoarthritis Outcome Score (HOOS) [83, 84]: the HOOS consists of five subscales including (1) pain, (2) symptoms and stiffness, (3) activities of daily living, (4) sport and recreation and (5) quality of life. Participants respond to a 5-point Likert scale for each subscale, and the scores are converted to a 101-point scale, with 100 indicating the best possible score (no difficulty), and 0 indicating extreme symptoms. The HOOS questionnaire is considered reliable ( $\mathrm{ICC}_{2,1}$ ranging from 0.75 to 0.97) [84].

Pain thoughts and depressive symptoms Pain Catastrophizing Scale (PCS) [85]: the 13-item PCS aims to assess the participants' thoughts and feelings associated with their pain. Using a 5-point Likert scale, participants are asked to rate the degree to which they have thoughts or feelings related to each item (e.g. 'I worry all the time about whether the pain will end'). The scale is scored from 0 to 52 with higher scores indicating greater pain catastrophizing, and is associated with higher-intensity physical and emotional stress in response to pain [85]. The PCS is considered a reliable and valid measure of pain catastrophizing [85].

Table 1 Hip-muscle strength testing protocol

\begin{tabular}{|c|c|c|c|}
\hline Action & Participant position & Dynamometer placement & Moment arm \\
\hline Abduction & $\begin{array}{l}\text { Supine. Participant holds onto the side of the } \\
\text { plinth to stabilise }\end{array}$ & $5 \mathrm{~cm}$ proximal to the lateral malleolus & $\begin{array}{l}\text { Greater trochanter to } 5 \mathrm{~cm} \text { proximal to } \\
\text { lateral malleolus }\end{array}$ \\
\hline Adduction & $\begin{array}{l}\text { Supine. Contralateral hip and knee flexed, with } \\
\text { foot placed flat on the plinth. Participant holds } \\
\text { onto the side of the plinth to stabilise }\end{array}$ & $5 \mathrm{~cm}$ proximal to the medial malleolus & $\begin{array}{l}\text { Greater trochanter to } 5 \mathrm{~cm} \text { proximal to } \\
\text { medial malleolus, measured along the } \\
\text { lateral surface of the lower limb. }\end{array}$ \\
\hline Internal rotation & $\begin{array}{l}\text { Sitting over the edge of the plinth. Arms folded } \\
\text { across chest }\end{array}$ & $5 \mathrm{~cm}$ proximal to the lateral malleolus & $\begin{array}{l}\text { Lateral knee joint line to } 5 \mathrm{~cm} \text { proximal } \\
\text { lateral malleolus }\end{array}$ \\
\hline External rotation & $\begin{array}{l}\text { Sitting over the edge of the plinth. Arms folded } \\
\text { across chest }\end{array}$ & $5 \mathrm{~cm}$ proximal to the medial malleolus & $\begin{array}{l}\text { Medial knee joint line to } 5 \mathrm{~cm} \text { proximal } \\
\text { medial malleolus, measured along the } \\
\text { lateral surface of the lower limb }\end{array}$ \\
\hline Hip flexion & $\begin{array}{l}\text { Sitting over the edge of the plinth. Arms folded } \\
\text { across chest }\end{array}$ & $\begin{array}{l}5 \mathrm{~cm} \text { proximal to the proximal border of } \\
\text { the patella }\end{array}$ & $\begin{array}{l}\text { Greater trochanter to } 5 \mathrm{~cm} \text { proximal to } \\
\text { proximal border of patella, measured } \\
\text { along the lateral surface of the lower } \\
\text { limb }\end{array}$ \\
\hline Hip extension & Prone. Testing knee flexed to $90^{\circ}$ & Calcaneus & Greater trochanter to Lateral joint line \\
\hline
\end{tabular}

Note: Resistance applied by the research assistant, with the exception of flexion and extension, where resistance is applied by a belt securing the dynamometer to an external anchor 
Brief Fear of Movement Scale for osteoarthritis (BFOM) [86]: adapted from the Tampa Scale of Kinesiophobia [87], the BFOM aims to assess the degree to which participants with osteoarthritis feel that physical movement will cause pain, injury or re-injury [86]. The six-item scale is scored from 0 to 24 , with a higher score indicating lower fear of movement (better score).

Patient Health Questionnaaire-9 (PHQ-9) [88]: the PHQ-9 is a nine-item valid and reliable scale that aims to measure depression severity. Scored from 0 to 27, participants can be classified as having mild $(\geq 5)$, moderate $(\geq 10)$, moderately severe $(\geq 15)$ and severe $(\geq 20)$ depressive symptoms.

Quality of life Assessment of Quality of Life Questionnaire (AQoL-8D) [89, 90]: the AQoL-8D contains 35 items that assess dimensions of quality of life related to independent living, happiness, mental health, coping, relationships, self-worth, pain and senses. It is a reliable and valid tool [90] that can produce a weighted utility score (0-1), or an unweighted, health-related quality of life score (0-100) with higher scores representing better quality of life.

Global change Global Rating of Change (GROC) [91]: the GROC consists of an 11-point Likert scale that asks participants to rate their perceived overall change in condition of their hip from the beginning of the study. A version of the GROC used in a previous study on hip pain [92] has been adapted for use in this study. A score of ' 0 ' will equate to 'no change'. If 'better/worse', participants will be asked to rate the degree of change from 'slightly better/worse' to 'very much better/worse', with scores ranging from +1 to +5 for 'better' and -1 to -5 for 'worse'. Scores will further be dichotomised to define 'success' as those with a score of 'moderately better' to 'very much better' (i.e. +3 or more).

Gluteal-muscle activity Muscle function of the gluteal muscles will be assessed with an EMG in a subset of 30 participants. Fine-wire electrodes will be inserted into GMed and GMin using previously validated procedures [93, 94]. Muscle activity will be recorded during six walking trials across a 10-m pathway. Amplitude and timing variables will be determined during the stance phase of the gait cycle as reported previously [33, 95].

Gluteal-muscle size and adiposity Muscle volume and adiposity will be assessed with MRI in a subset of 30 participants (the same participants undergoing EMG testing). Muscles of interest will include GMin, GMed and gluteus maximus, as well as tensor fascia lata. Muscle volume and adiposity will be determined through off-line processing of de-identified images using customised MATLAB software (The MathWorks, Inc., Natick, MA, USA) [96]. Axial slices of each muscle will be traced to establish cross-sectional area, and multiplied by slice thickness to calculate volume [26, 97]. To determine muscle adiposity, a Muscle-Fat Index (MFI) will be calculated from the axial slices as the proportion of adipose tissue to total muscle (fat/fat + muscle) [98]. This technique has been validated against the 'gold standard', biopsy [99]. Given the segmental nature of GMin and GMed, a MFI will be calculated for anterior and posterior regions of each muscle [96].

\section{Trial follow-up}

The primary time-point will be at the completion of the intervention (13 weeks). Additional measures of some outcomes (questionnaires) will be recorded at 7 and 25 weeks. The schedule of enrolment, assessment, intervention and follow-up can be seen in Fig. 2 .

\section{Success of blinding}

The success of participant blinding will be assessed 1 week after commencing the intervention by having participants nominate which intervention they feel they have been allocated to ('generalised exercise programme' or 'targeted gluteal programme' or 'unsure'. It is not anticipated that large differences in outcome would be present 1 week after commencing the intervention, so the potential benefit (or otherwise) of the intervention will not influence their decision. The validity of assessing the effectiveness of blinding for interventions that may expect large effect sizes is questionable, and is now no longer a mandatory component of the Consolidated Standards of Reporting Trials (CONSORT) Statement $[100,101]$.

\section{Adverse events}

Adverse events will be monitored and recorded by the physiotherapist and participant. These may include minor aches and pains through to serious adverse events (e.g. myocardial infarction) associated with beginning a new exercise programme.

\section{Sample size and power analysis}

The minimal clinically important difference for the WOMAC is 6 physical function units [72]. Using data from the sham intervention of a previous RCT in people with hip osteoarthritis [21], the standard deviation (SD) of change in the WOMAC over 13 weeks is 9.2 units, assuming a baseline to follow-up correlation of 0.60 . A total sample size of 76 participants (38 per group) is required to detect a clinically important difference between sham and intervention groups, with a power of $80 \%$ and an alpha of 0.05 . A clinically important difference of 6 units between groups, assuming a 


\begin{tabular}{|c|c|c|c|c|c|c|c|c|}
\hline CONSTRUCT & & STUDY PERIOD & & & & & & \\
\hline & & Enrolment & Allocation & & & & & \\
\hline & TIMEPOINT: & \begin{tabular}{|l|} 
Approximately \\
one week \\
before starting \\
the intervention
\end{tabular} & $\begin{array}{l}\text { Approximately one } \\
\text { day prior to } \\
\text { starting the } \\
\text { intervention. Trial } \\
\text { physiotherapist } \\
\text { opens envelope to } \\
\text { reveal allocation }\end{array}$ & $\begin{array}{c}0 \\
\text { weeks }\end{array}$ & $\begin{array}{c}7 \\
\text { weeks }\end{array}$ & $\begin{array}{c}12 \\
\text { weeks }\end{array}$ & $\begin{array}{c}{ }^{\mathrm{a}} 13 \\
\text { weeks }\end{array}$ & $\begin{array}{c}25 \\
\text { weeks }\end{array}$ \\
\hline & ENROLMENT: & & & & & & & \\
\hline & Eligibility screen & $\mathrm{x}$ & & & & & & \\
\hline & Informed consent & $\mathrm{x}$ & & & & & & \\
\hline & Randomisation Allocation & & $x$ & & & & & \\
\hline & & & & & & & & \\
\hline & INTERVENTIONS: & & & & & & & \\
\hline & GHOst & & & 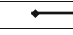 & & $\vec{\longrightarrow}$ & & \\
\hline & Sham & & & 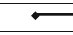 & & $\longrightarrow$ & & \\
\hline & ASSESSMENTS. & & & & & & & \\
\hline $\begin{array}{l}\text { Physical } \\
\text { function- self } \\
\text { reported }\end{array}$ & $\begin{array}{l}\text { "WOMAC- physical } \\
\text { function [70] }\end{array}$ & $x$ & & & $\mathrm{x}$ & & $\mathrm{x}$ & $x$ \\
\hline Physical activity & Accelerometry & $\mathrm{x}$ & & & & & $\mathrm{x}$ & \\
\hline $\begin{array}{l}\text { Physical } \\
\text { function }\end{array}$ & $\begin{array}{l}\text { OARSI recommended tests } \\
\text { [69] } \\
\text { - } 40 \mathrm{~m} \text { fast paced } \\
\text { - } \text { walk test } \\
\text { - Stair climb test } \\
\text { - Chair stand test }\end{array}$ & $x$ & & & & & $x$ & \\
\hline $\begin{array}{l}\text { Physical } \\
\text { activity- self } \\
\text { reported }\end{array}$ & IPAQ [78] last 7 day recall & $x$ & & & $x$ & & $x$ & $x$ \\
\hline Strength & $\begin{array}{l}\text { Isometric hip strength } \\
\text { tests- HHD }\end{array}$ & $x$ & & & & & $x$ & \\
\hline Pain & $\begin{array}{l}\text { Numerical rating scale- self } \\
\text { report of average pain } \\
\text { over the last week [82] }\end{array}$ & $x$ & & & $x$ & & $x$ & $x$ \\
\hline $\begin{array}{l}\text { Hip Specific } \\
\text { Global Outcome } \\
\text { Measures } \\
\end{array}$ & HOOS [83] & $x$ & & & $x$ & & $x$ & $x$ \\
\hline Quality of Life & AQoL [89] & $\mathrm{x}$ & & & $\mathrm{x}$ & & $\mathrm{x}$ & $\mathrm{x}$ \\
\hline \multirow[t]{3}{*}{ Pain thoughts } & \begin{tabular}{|l|} 
Pain Catastrophizing Scale \\
{$[85]$}
\end{tabular} & $x$ & & & $x$ & & $\mathrm{x}$ & $\mathrm{x}$ \\
\hline & $\begin{array}{l}\text { Brief fear of movement } \\
\text { scale for osteoarthritis [86] }\end{array}$ & $x$ & & & $x$ & & $x$ & $x$ \\
\hline & \begin{tabular}{|l|} 
Patient Health \\
Questionnaaire-9 [88]
\end{tabular} & $\mathrm{x}$ & & & $x$ & & $\mathrm{x}$ & $x$ \\
\hline Global Change & $\begin{array}{l}\text { Global rating of change } \\
\text { scale [91] }\end{array}$ & & & & $\mathrm{x}$ & & $\mathrm{x}$ & $\mathrm{x}$ \\
\hline \multicolumn{9}{|c|}{ Additional measures in a subset of 30 participants only } \\
\hline $\begin{array}{l}\text { Gluteal muscle } \\
\text { activity }\end{array}$ & $\begin{array}{l}\text { Gluteus minimus and } \\
\text { gluteus medius EMG in } \\
\text { gait [93] }\end{array}$ & $x$ & & & & & $\mathrm{x}$ & \\
\hline $\begin{array}{l}\text { Gluteal muscle } \\
\text { size and } \\
\text { adiposity }\end{array}$ & $\begin{array}{l}\text { MRI volume [26] and } \\
\text { adiposity [96] }\end{array}$ & $x$ & & & & & $x$ & \\
\hline
\end{tabular}

Fig. 2 Standard Protocol Items: Recommendations for Interventional Trials (SPIRIT) Checklist: patient schedule of procedures

within-group SD of 9.2 units equates to a moderate to large effect size (0.65). To account for potential drop-outs similar to those reported in previous studies [21] we will recruit 90 participants (45 per group).

\section{Statistical analysis}

Statistical analysis will be using intention-to-treat principles and on a per-protocol basis. Data analysed will focus on detecting the between-group treatment and within-group treatment effects (with effect sizes and 95\% confidence intervals) at each of the follow-up time-points. A linear mixed model will be used for the primary analysis of the changes in the WOMAC due to its advantages in modelling the influence of nonlinear, individual differences over time. Adjustments will be made for the respective baseline outcome measures, as well as age, sex and BMI; and adjustments for other baseline variables that have evidence of imbalances between groups (e.g. radiographic severity). The secondary outcomes will be assessed using $t$ tests or Mann-Whitney $U$ tests, adjusting for baseline differences if required. The Global Rating of Change Scale will be dichotomised to define 'success' as those with a score of 'moderately better' to 'very much better' (i.e. +3 or more). A generalised 
mixed model (adjusted for baseline differences and covariates) will be used to assess differences in the proportion of 'successes' between groups over each time-point.

\section{Discussion}

This RCT will be the first to investigate the effect of a targeted gluteal rehabilitation programme compared to a sham exercise programme for improving physical function in people with hip osteoarthritis. The targeted GHOst protocol is a structured exercise programme that incorporates gait retraining, motor control and high-intensity resistance exercises. It requires minimal equipment and is implemented by community physiotherapists. This pragmatic design could enable easy integration into any physiotherapy clinical setting. The research design aims to minimise bias associated with selection, measurement and confounding. The results of this study will have significant implications in terms of maximising physical function in people with hip osteoarthritis which may ultimately affect their quality of life.

The GHOst intervention has been specifically developed for people with hip osteoarthritis. Selection of a sample of people with hip osteoarthritis is, therefore, critical to the outcome of this study. We have used an accepted criteria for hip osteoarthritis diagnosis that includes clinical and radiographic confirmation [44]. More recent criteria, for example from the National Institute for Health and Care Excellence (NICE) [13], suggests a clinical diagnosis without the need for radiographic confirmation. Such criteria would enhance generalisability and potentially maximise recruitment rates into the study. However, clinical diagnoses without radiographic confirmation are often paired with higher age thresholds (NICE guidelines; $>45$ years) to improve the specificity of the diagnosis. It is increasingly being recognised that osteoarthritis is not just an 'old-persons" disease [102]. A large increase in prevalence of osteoarthritis has been observed in Canadians aged over 35 years (1994-2002 data) [103], and the projected change in healthcare costs associated with osteoarthritis in Australia in those under 65 years is greatest in the 35-44 years age bracket [7]. Radiographic confirmation would, therefore, allow for inclusion of younger people with osteoarthritis, who may have a significant impact on the burden of disease in years to come.

Measurement error and confounding have been limited through the study design. All assessors are blinded to group allocation, and our primary outcomes are valid and reliable. A unique element of the current study is the inclusion of secondary outcomes such as muscle activity (EMG) and muscle size (MRI), which will provide mechanistic evidence of differences (or lack of) between interventions. It is not possible to blind the therapists to group allocation, and this may confound the effects of the intervention if therapists deliver the GHOst intervention with more enthusiasm than the sham. This could potentially lead to inflated effect sizes in favour of the intervention. This will be minimised with appropriate training for therapists on delivery of both interventions with equal enthusiasm.

The results of this study will be disseminated via journal publication and conference presentations. Participants will receive a summary of results via email at the completion of the study. The interventions have been designed to facilitate translation into clinical practice and will aid health and medical practitioners with evidence-based decisionmaking for the non-pharmacological management of hip osteoarthritis.

\section{Trial status}

This is protocol version 3 (20 October 2017). Recruitment commenced 26 July 2017. Recruitment is expected to be completed by 1 June 2019.

\section{Additional files}

Additional file 1: Standard Protocol Items: Recommendations for Interventional Trials (SPIRIT) 2013 Checklist: recommended items to address in a clinical trial protocol and related documents*. (DOC $135 \mathrm{~kb}$ )

Additional file 2: Flow diagram illustrating a clinical diagnosis of gluteal tendinopathy that would warrant exclusion from the proposed study [49]. Abbreviations: FABER- flexion, abduction, external rotation test, $O A$ osteoarthritis. (DOCX $40 \mathrm{~kb}$ )

Additional file 3: Flow diagram illustrating screening for lumbar pathology as the source of hip pain. Abbreviations: $p S L R$ passive straight leg raise, performed to $45^{\circ}$ of hip flexion. (DOCX $25 \mathrm{~kb}$ )

Additional file 4: Example of the participant information and consent form. Bendigo site. (PDF $184 \mathrm{~kb}$ )

\section{Abbreviations}

ANZCTR: Australian and New Zealand Clinical Trials Registry; AQoL8D: Assessment of Quality of Life Questionnaire-8D; BFOM: Brief Fear of Movement Scale for Osteoarthritis; BMI: Body Mass Index; CONSORT: Consolidated Standards of Reporting Trials;

EMG: Electromyography; FABER: Flexion, abduction, external rotation test; GHOst: Gluteal exercises for Hip Osteoarthritis; GMed: Gluteus medius; GMin: Gluteus minimus; GROC: Global Rating of Change; HDEC: Health and Disability Ethics Committee; HOOS: Hip Osteoarthritis Outcome Score; HREC: Human Research Ethics Committee; MFI: Muscle-Fat Index; MRI: Magnetic resonance imaging; MVPA: Moderate to vigorous physical activity; NICE: National Institute for Health and Care Excellence; NRS: Numerical rating scale; NSAIDS: Non-steroidal anti-inflammatory drugs; PCS: Pain Catastrophizing Scale; PHQ-9: Patient Health Questionnaire-9; SD: Standard deviation; SPIRIT: Standard Protocol Items: Recommendations for Interventional Trials; UTN: Universal Trial Number; VAS: Visual analogue scale; WOMAC: Western Ontario and McMaster Universities Osteoarthritis Index

\section{Acknowledgements}

We wish to acknowledge in advance the treating physiotherapists and the participants in Melbourne, Bendigo, Brisbane and Dunedin for their involvement in this study.

\section{Funding}

RG, AS, TP, SW, AZ, MK received funding from Arthritis Australia (Zimmer Biomet Australia Grant; peer reviewed), Arthritis New Zealand (R273, peer- 
reviewed) and La Trobe University (Research Focus Area for Sport, Exercise and Rehabilitation Project Grant, peer-reviewed). Funding bodies were not involved in the design, collection, analysis and interpretation of data; in the writing of the manuscript; or in the decision to submit the manuscript for publication

\section{Availability of data and materials}

The datasets used and/or analysed during the current study will be available from the corresponding author on reasonable request.

\section{Authors' contributions}

AS and TP developed the GHOst exercise intervention. RAG, AS, TP, SW, AZ and MK approved the final version of the GHOst exercise intervention. RAG AS, TP, SW, AZ and MK designed the study. AS drafted the protocol manuscript. All authors have read and approved the final manuscript. RAG, AS, TP, SW, AZ and MK obtained funding for the study.

\section{Competing interests}

The authors declare that they have no competing interests.

\section{Ethics approval and consent to participate}

Ethics approval has been provided by the Bendigo Health Care Group Human Research Ethics Committee (HREC/17/BHCG/3); La Trobe University HREC; The University of Queensland (20,170,001,541/HREC/17/BHCG/3) and the Otago University HDEC (17/STH/205).

\section{Consent for publication}

Not applicable.

\section{Publisher's Note}

Springer Nature remains neutral with regard to jurisdictional claims in published maps and institutional affiliations.

\section{Author details}

'Department of Rehabilitation, Nutrition and Sport; La Trobe University, Bundoora, VIC, Australia. ${ }^{2}$ School of Health and Rehabilitation Sciences, The University of Queensland, St Lucia, QLD, Australia. ${ }^{3}$ Department of Anatomy, School of Biomedical Sciences, University of Otago, Dunedin, New Zealand. ${ }^{4}$ Department of Pharmacy and Applied Science, La Trobe University, Bendigo, VIC, Australia. ${ }^{5}$ Exercise Physiology, La Trobe Rural Health School, La Trobe University, Bendigo, VIC, Australia.

\section{Received: 4 May 2018 Accepted: 23 August 2018}

\section{Published online: 20 September 2018}

\section{References}

1. Global Burden of Disease Study - 2016 Results. http://vizhub.healthdata.org/ gbd-compare/. Accessed Mar 2018.

2. Busija $L$, Bridgett $L$, Williams SRM, Osborne $R H$, Buchbinder R, March L, Fransen M. Osteoarthritis. Best Pract Res Clin Rheumatol. 2010;24(6):757-68.

3. van Dijk GM, Dekker J, Veenhof $\mathrm{C}$, van den Ende $\mathrm{CH}$. Course of functional status and pain in osteoarthritis of the hip or knee: a systematic review of the literature. Arthritis Care Res. 2006;55(5):779-85.

4. Pisters MF, Veenhof C, van Dijk GM, Dekker J. Avoidance of activity and limitations in activities in patients with osteoarthritis of the hip or knee: a 5year follow-up study on the mediating role of reduced muscle strength. Osteoarthr Cartil. 2014;22(2):171-7.

5. Wallis JA, Webster KE, Levinger $P$, Taylor NF. What proportion of people with hip and knee osteoarthritis meet physical activity guidelines? A systematic review and meta-analysis. Osteoarthr Cartil. 2013;21(11):1648-59.

6. Ackerman IN, Bucknill A, Page RS, Broughton NS, Roberts C, Cavka B, Schoch $P, B$ rand $C A$. The substantial personal burden experienced by younger people with hip or knee osteoarthritis. Osteoarthr Cartil. 2015;23(8):1276-84.

7. Ackerman I, Bohensky M, Pratt C, Gorelik A, Liew D. Counting the cost part 1: healthcare costs: the current and future burden of arthritis. Sydney: Arthritis Australia; 2016.

8. Cross M, Smith E, Hoy D, Nolte S, Ackerman I, Fransen M, Bridgett L Williams S, Guillemin F, Hill CL. The global burden of hip and knee osteoarthritis: estimates from the Global Burden of Disease 2010 study. Ann Rheum Dis. 2014;73(7):1323-30.
9. Brosseau L, Taki J, Desjardins B, Thevenot O, Fransen M, Wells GA, Mizusak Imoto A, Toupin-April K, Westby M, Álvarez Gallardo IC. The Ottawa panel clinical practice guidelines for the management of knee osteoarthritis. Part two: strengthening exercise programs. Clin Rehabil. 2017;31(5):596-611.

10. Brosseau L, Wells GA, Pugh AG, Smith CA, Rahman P, Àlvarez Gallardo IC, Toupin-April K, Loew L, De Angelis G, Cavallo S. Ottawa panel evidencebased clinical practice guidelines for therapeutic exercise in the management of hip osteoarthritis. Clin Rehabil. 2016;30(10):935-46.

11. Cibulka MT, Bloom NJ, Enseki KR, MacDonald CW, Woehrle J, McDonough CM. Hip pain and mobility deficits—hip osteoarthritis: revision 2017: clinical practice guidelines linked to the international classification of functioning, disability and health from the orthopaedic section of the American Physical Therapy Association. J Orthop Sports Phys Ther. 2017;47(6):A1-A37.

12. McAlindon TE, Bannuru RR, Sullivan MC, Arden NK, Berenbaum F, BiermaZeinstra SM, Hawker GA, Henrotin Y, Hunter DJ, Kawaguchi H, et al. OARSI guidelines for the non-surgical management of knee osteoarthritis. Osteoarthr Cartil. 2014;22(3):363-88.

13. National Institute for Health and Clinical Excellence. Osteoarthritis: care and management. London: NICE; 2014.

14. Zacharias A, Green RA, Semciw Al, Kingsley MIC, Pizzari T. Efficacy of rehabilitation programs for improving muscle strength in people with hip or knee osteoarthritis: a systematic review with meta-analysis. Osteoarthr Cartil. 2014;22(11):1752-73.

15. Hunter DJ, Eckstein F. Exercise and osteoarthritis. J Anat. 2009;214(2): 197-207.

16. Blazek AD, Nam J, Gupta R, Pradhan M, Perera P, Weisleder NL, Hewett TE, Chaudhari AM, Lee BS, Leblebicioglu B, et al. Exercise-driven metabolic pathways in healthy cartilage. Osteoarthr Cartil. 2016;24(7):1210-22.

17. Bennell K. Physiotherapy management of hip osteoarthritis. J Phys. 2013; 59(3):145-57.

18. Bennell KL, Dobson F, Roos EM, Skou ST, Hodges P, Wrigley TV, Kyriakides M, Metcalf B, Hunt MA, Hinman RS. Influence of Biomechanical Characteristics on Pain and Function Outcomes From Exercise in Medial Knee Osteoarthritis and Varus Malalignment: Exploratory Analyses From a Randomized Controlled Trial. Arthritis Care Res. 2015;67(9):1281-1288.

19. Fransen M, McConnell S, Harmer AR, Van der Esch M, Simic M, Bennell KL. Exercise for osteoarthritis of the knee. Cochrane Database Syst Rev. 2015;1: CD004376.

20. Fransen M, McConnell S, Hernandez-Molina G, Reichenbach S. Exercise for osteoarthritis of the hip. Cochrane Database Syst Rev. 2014(4):CD007912.

21. Bennell KL, Egerton T, Martin J, Abbott JH, Metcalf B, McManus F, Sims K, Pua Y-H, Wrigley TV, Forbes A. Effect of physical therapy on pain and function in patients with hip osteoarthritis: a randomized clinical trial. JAMA. 2014;311(19):1987-97.

22. Bennell $\mathrm{KL}$, Buchbinder R, Hinman RS. Physical therapies in the management of osteoarthritis: current state of the evidence. Curr Opin Rheumatol. 2015:27(3):304-11.

23. Bennell $\mathrm{KL}$, Hinman RS. A review of the clinical evidence for exercise in osteoarthritis of the hip and knee. J Sci Med Sport. 2011;14(1):4-9.

24. Hall M, Allison K, Wrigley TV, Metcalf BR, Pua YH, Van Ginckel A, Bennell KL. Frontal plane hip joint loading according to pain severity in people with hip osteoarthritis. J Orthop Res. 2018;36(6):1637-1644.

25. Zacharias A, Green RA, Semciw A, English DJ, Kapakoulakis T, Pizzari T. Atrophy of hip abductor muscles is related to clinical severity in a hip osteoarthritis population. Clin Anat. 2018;31(4):507-13.

26. Zacharias A, Pizzari T, English DJ, Kapakoulakis T, Green RA. Hip abductor muscle volume in hip osteoarthritis and matched controls. Osteoarthr Cartil. 2016;24(10):1727-35

27. Constantinou M, Loureiro A, Carty C, Mills P, Barrett R. Hip joint mechanics during walking in individuals with mild-to-moderate hip osteoarthritis. Gait Posture. 2017;53(Suppl C):162-7.

28. Constantinou M, Barrett R, Brown M, Mills P. Spatial-temporal gait characteristics in individuals with hip osteoarthritis: a systematic literature review and meta-analysis. J Orthop Sports Phys Ther. 2014; 44(4):291-B297.

29. King MG, Lawrenson PR, Semciw Al, Middleton KJ, Crossley KM. Lower limb biomechanics in femoroacetabular impingement syndrome: a systematic review and meta-analysis. Br J Sports Med. 2018:52(9):566-80.

30. Hurwitz D, Hulet C, Andriacchi T, Rosenberg A, Galante J. Gait compensations in patients with osteoarthritis of the hip and their relationship to pain and passive hip motion. J Orthop Res. 1997;15(4):629-35. 
31. Semciw Al. It's not just the size that counts, but how you use it: research into the anterior gluteus minimus. Sport Health. 2014;32(1):50-5.

32. Ganderton C, Pizzari T, Harle T, Cook J, Semciw A. A comparison of gluteus medius, gluteus minimus and tensor facia latae muscle activation during gait in post-menopausal women with and without greater trochanteric pain syndrome. J Electromyogr Kinesiol. 2017;33:39-47.

33. Semciw Al, Green RA, Murley GS, Pizzari T. Gluteus minimus: an intramuscular EMG investigation of anterior and posterior segments during gait. Gait Posture. 2014;39(2):822-6.

34. Lewis $\mathrm{CL}$, Sahrmann SA, Moran DW. Anterior hip joint force increases with hip extension, decreased gluteal force, or decreased iliopsoas force. J Biomech. 2007;40(16):3725-31.

35. Safran MR, Giordano G, Lindsey DP, Gold GE, Rosenberg J, Zaffagnini S, Giori NJ. Strains across the acetabular labrum during hip motion: a cadaveric model. Am J Sports Med. 2011:39(1 suppl):92S-102S.

36. Grimaldi A, Richardson C, Stanton W, Durbridge G, Donnelly W, Hides J. The association between degenerative hip joint pathology and size of the gluteus medius, gluteus minimus and piriformis muscles. Manual Ther. 2009; 14(6):605-10.

37. Kivle K, Lindland E, Mjaaland KE, Pripp AH, Svenningsen S, Nordsletten L. The gluteal muscles in end-stage osteoarthritis of the hip: intra- and interobserver reliability and agreement of MRI assessments of muscle atrophy and fatty degeneration. Clin Radiol 2018;73(7):675.e617-675.e624.

38. Kawasaki M, Hasegawa Y, Okura T, Ochiai S, Fujibayashi T. Muscle damage after total hip arthroplasty through the direct anterior approach for developmental dysplasia of the hip. J Arthroplast. 2017; 32(8):2466-73.

39. Pfirrmann CW, Notzli HP, Dora C, Hodler J, Zanetti M. Abductor tendons and muscles assessed at MR imaging after total hip arthroplasty in asymptomatic and symptomatic patients. Radiology. 2005;235(3):969-76.

40. Moher D, Hopewell S, Schulz KF, Montori V, Gøtzsche PC, Devereaux P, Elbourne D, Egger M, Altman DG. CONSORT 2010 explanation and elaboration: updated guidelines for reporting parallel group randomised trials. J Clin Epidemiol. 2010;63(8):e1-e37.

41. Chan A-W, Tetzlaff JM, Altman DG, Laupacis A, Gøtzsche PC, Krleža-Jerić K, Hróbjartsson A, Mann H, Dickersin K, Berlin JA. SPIRIT 2013 statement: defining standard protocol items for clinical trials. Ann Intern Med. 2013; 158(3):200-7.

42. Chan A-W, Tetzlaff JM, Gøtzsche PC, Altman DG, Mann H, Berlin JA, Dickersin K, Hróbjartsson A, Schulz KF, Parulekar WR. SPIRIT 2013 explanation and elaboration: guidance for protocols of clinical trials. BMJ. 2013;346:e7586.

43. Lane NE, Hochberg MC, Nevitt MC, Simon LS, Nelson AE, Doherty M, Herontin Y, Flechsenhar K. OARSI clinical trials recommendations: design and conduct of clinical trials for hip osteoarthritis. Osteoarthr Cartil. 2015; 23(5):761-71.

44. Altman R, Alarcon G, Appelrouth D, Bloch D, Borenstein D, Brandt K, Brown C, Cooke T, Daniel W, Feldman D. The American College of Rheumatology criteria for the classification and reporting of osteoarthritis of the hip. Arthritis Rheumatol. 1991;34(5):505-14.

45. Kellgren J, Lawrence J. Radiological assessment of osteo-arthrosis. Ann Rheum Dis. 1957:16(4):494.

46. Murray D, Fitzpatrick R, Rogers K, Pandit H, Beard D, Carr A, Dawson J. The use of the Oxford Hip and Knee Scores. Bone Joint J. 2007;89(8):1010-4.

47. Martinelli N, Longo UG, Marinozzi A, Franceschetti E, Costa V, Denaro V. Cross-cultural adaptation and validation with reliability, validity, and responsiveness of the Italian version of the Oxford Hip Score in patients with hip osteoarthritis. Qual Life Res. 2011;20(6):923-9.

48. Hubley-Kozey C, Deluzio K, Landry S, McNutt J, Stanish W. Neuromuscular alterations during walking in persons with moderate knee osteoarthritis. J Electromyogr Kinesiol. 2006;16(4):365-78.

49. Ganderton C, Semciw Al, Cook J, Pizzari T. Demystifying the clinical diagnosis of greater trochanteric pain syndrome in women. J Women's Health. 2017;26(6):633-43.

50. Fearon AM, Scarvell JM, Neeman T, Cook JL, Cormick W, Smith PN. Greater trochanteric pain syndrome: defining the clinical syndrome. $\mathrm{Br} J$ Sports Med. 2013:47(10):649-53.

51. Grimaldi A, Mellor R, Nicolson P, Hodges P, Bennell K, Vicenzino B. Utility of clinical tests to diagnose MRI-confirmed gluteal tendinopathy in patients presenting with lateral hip pain. Br J Sports Med. 2017;51(6):519-524.

52. Frost $R$, Levati $S, M c C l u r g ~ D$, Brady M, Williams B. What adherence measures should be used in trials of home-based rehabilitation interventions? A systematic review of the validity, reliability, and acceptability of measures. Arch Phys Med Rehabil. 2017:98(6):1241-1256.e1245.

53. Watelain E, Dujardin F, Babier F, Dubois D, Allard P. Pelvic and lower limb compensatory actions of subjects in an early stage of hip osteoarthritis. Arch Phys Med Rehabil. 2001;82(12):1705-11.

54. Nascimento LR, de Oliveira CQ, Ada L, Michaelsen SM, Teixeira-Salmela LF. Walking training with cueing of cadence improves walking speed and stride length after stroke more than walking training alone: a systematic review. J Phys. 2015;61(1):10-5.

55. Hoogkamer W, Meyns P, Duysens J. Steps forward in understanding backward gait: from basic circuits to rehabilitation. Exerc Sport Sci Rev. 2014; 42(1):23-9.

56. Yang Y-R, Yen J-G, Wang R-Y, Yen L-L, Lieu F-K. Gait outcomes after additional backward walking training in patients with stroke: a randomized controlled trial. Clin Rehabil. 2005:19(3):264-73.

57. Lewis CL, Ferris DP. Walking with increased ankle pushoff decreases hip muscle moments. J Biomech. 2008:41(10):2082-9.

58. Lewis $\mathrm{CL}$, Garibay EJ. Effect of increased pushoff during gait on hip joint forces. J Biomech. 2015;48(1):181-5.

59. Semciw Al. It's not just the size that counts, but how you use it. SportsPhysio. 2014;1:24-6.

60. Ganderton C, Pizzari T, Cook J, Semciw A. A simple hip exercise: hip hitching elicits high levels of deep gluteal muscle activity. J Sci Med Sport. 2015;19:e18.

61. Ganderton C, Semciw A, Cook J, Pizzari T. Does menopausal hormone therapy (MHT), exercise or a combination of both, improve pain and function in post-menopausal women with greater trochanteric pain syndrome (GTPS)? A randomised controlled trial. BMC Women's Health. 2016;16(1):1.

62. Ganderton C, Pizzari T, Cook J, Semciw A. Gluteus minimus and gluteus medius muscle activity during common rehabilitation exercises in healthy postmenopausal women. J Orthop Sports Phys Ther. 2017;47(12):914-22.

63. Moore D, Semciw Al, McClelland J, Wajswelner H, Pizzari T. Rehabilitation exercises for the gluteus minimus muscle segments - an electromyography study. J Sport Rehabil. 2018:1-26.

64. Borg GA. Psychophysical bases of perceived exertion. Med Sci Sports Exerc. 1982;14(5):377-81.

65. Andersen LL, Andersen CH, Mortensen OS, Poulsen OM, Biørnlund IBT, Zebis MK. Muscle activation and perceived loading during rehabilitation exercises: comparison of dumbbells and elastic resistance. Phys Ther. 2010;90(4):538-49.

66. Brandt M, Jakobsen MD, Thorborg K, Sundstrup E, Jay K, Andersen LL. Perceived loading and muscle activity during hip strengthening exercises: comparison of elastic resistance and machine exercises. Int J Sports Phys Ther. 2013;8(6):811.

67. Day ML, Mcguigan MR, Brice G, Foster C. Monitoring exercise intensity during resistance training using the Session RPE Scale. J Strength Cond Res. 2004;18(2):353-8.

68. Main C, Sowden G, Hill J, Watson P, Hay E. Integrating physical and psychological approaches to treatment in low back pain: the development and content of the STarT Back trial's 'high-risk'intervention (StarT Back; ISRCTN 37113406). Physiotherapy. 2012;98(2):110-6.

69. Dobson F, Hinman RS, Roos EM, Abbott JH, Stratford P, Davis AM, Buchbinder R, Snyder-Mackler L, Henrotin Y, Thumboo J, et al. OARSI recommended performance-based tests to assess physical function in people diagnosed with hip or knee osteoarthritis. Osteoarthritis Cartilage. 2013;21(8):1042-1052.

70. Bellamy N, Buchanan WW, Goldsmith CH, Campbell J, Stitt LW. Validation study of WOMAC: a health status instrument for measuring clinically important patient relevant outcomes to antirheumatic drug therapy in patients with osteoarthritis of the hip or knee. J Rheumatol. 1988;15(12):1833-40.

71. McConnell S, Kolopack P, Davis AM. The Western Ontario and McMaster Universities Osteoarthritis Index (WOMAC): a review of its utility and measurement properties. Arthritis Care Res. 2001;45(5):453-61.

72. Angst F, Aeschlimann A, Stucki G. Smallest detectable and minimal clinically important differences of rehabilitation intervention with their implications for required sample sizes using WOMAC and SF-36 quality of life measurement instruments in patients with osteoarthritis of the lower extremities. Arthritis Care Res. 2001:45(4):384-91.

73. McClain JJ, Sisson SB, Tudor-Locke C. Actigraph accelerometer interinstrument reliability during free-living in adults. Med Sci Sports Exerc. 2007;39(9):1509-14. 
74. Evenson KR, Buchner DM, Morland KB. Objective measurement of physical activity and sedentary behavior among US adults aged 60 years or older. Prev Chronic Dis. 2012;9:E26

75. Sasaki JE, John D, Freedson PS. Validation and comparison of ActiGraph activity monitors. J Sci Med Sport. 2011;14(5):411-6.

76. Migueles JH, Cadenas-Sanchez C, Ekelund U, Nyström CD, Mora-Gonzalez J, Löf M, Labayen I, Ruiz JR, Ortega FB. Accelerometer data collection and processing criteria to assess physical activity and other outcomes: a systematic review and practical considerations. Sports Med. 2017:47(9):1821-45.

77. Dobson F, Hinman RS, Hall M, Marshall CJ, Sayer T, Anderson C, Newcomb N, Stratford PW, Bennell KL. Reliability and measurement error of the Osteoarthritis Research Society International (OARSI) recommended performance-based tests of physical function in people with hip and knee osteoarthritis. Osteoarthr Cartil. 2017;25(11):1792-6

78. Craig CL, Marshall AL, Sjöström M, Bauman AE, Booth ML, Ainsworth BE, Pratt M, Ekelund U, Yngve A, Sallis JF. International Physical Activity Questionnaire: 12-country reliability and validity. Med Sci Sports Exerc. 2003; 35(8):1381-95.

79. Thorborg K, Bandholm T, Hölmich P. Hip-and knee-strength assessments using a hand-held dynamometer with external belt-fixation are inter-tester reliable. Knee Surg Sports Traumatol Arthrosc. 2013;21(3):550-5.

80. Thorborg K, Petersen J, Magnusson S, Hölmich P. Clinical assessment of hip strength using a hand-held dynamometer is reliable. Scand J Med Sci Sports. 2010;20(3):493-501.

81. Kemp JL, Schache AG, Makdissi M, Sims KJ, Crossley KM. Greater understanding of normal hip physical function may guide clinicians in providing targeted rehabilitation programmes. J Sci Med Sport. 2013; 16(4):292-6

82. Abbott JH, Schmitt J. Minimum important differences for the PatientSpecific Functional Scale, 4 region-specific outcome measures, and the numeric pain rating scale. J Orthop Sports Phys Ther. 2014;44(8):560-4.

83. Klässbo M, Larsson E, Mannevik E. Hip disability and Osteoarthritis Outcome Score an extension of the Western Ontario and McMaster Universities Osteoarthritis Index. Scand J Rheumatol. 2003;32(1):46-51.

84. Nilsdotter A, Bremander A. Measures of hip function and symptoms: Harris Hip Score (HHS), Hip disability and Osteoarthritis Outcome Score (HOOS), Oxford Hip Score (OHS), Lequesne Index of Severity for Osteoarthritis of the Hip (LISOH), and American Academy of Orthopedic Surgeons (AAOS) hip and knee questionnaire. Arthritis Care Res. 2011;63(S11):S200-7.

85. Sullivan MJ, Bishop SR, Pivik J. The Pain Catastrophizing Scale: development and validation. Psychol Assess. 1995;7(4):524.

86. Shelby RA, Somers TJ, Keefe FJ, DeVellis BM, Patterson C, Renner JB, Jordan JM. Brief Fear of Movement Scale for Osteoarthritis. Arthritis Care Res. 2012; 64(6):862-71.

87. Korri S, Miller R, Todd D. Kinesiophobia: a new view of chronic pain behaviour. Pain Manag. 1990;3:35-43.

88. Kroenke K, Spitzer RL, Williams JBW. The PHQ-9: validity of a brief depression severity measure. J Gen Intern Med. 2001;16(9):606-13.

89. Whitfield K, Buchbinder R, Segal L, Osborne RH. Parsimonious and efficient assessment of health-related quality of life in osteoarthritis research: validation of the Assessment of Quality of Life (AQoL) instrument. Health Qual Life Outcomes. 2006:4(1):19.

90. Richardson J, lezzi A, Khan MA, Maxwell A. Validity and reliability of the Assessment of Quality of Life (AQoL)-8D Multi-Attribute Utility Instrument. Patient. 2014;7(1):85-96.

91. Kamper SJ, Maher CG, Mackay G. Global Rating of Change scales: a review of strengths and weaknesses and considerations for design. J Manual Manipulat Therapy. 2009;17(3):163-70.

92. Mellor R, Grimaldi A, Wajswelner H, Hodges P, Abbott JH, Bennell K, Vicenzino B. Exercise and load modification versus corticosteroid injection versus 'wait and see' for persistent gluteus medius/minimus tendinopathy (the LEAP trial): a protocol for a randomised clinical trial. BMC Musculoskelet Disord. 2016;17(1):1-17.

93. Semciw Al, Green RA, Pizzari T, Briggs C. Verification of a standardized method for inserting intramuscular EMG electrodes into uniquely oriented segments of gluteus minimus and gluteus medius. Clin Anat. 2013;26(2):244-52.

94. Semciw Al, Pizzari T, Green RA. Technical application and the level of discomfort associated with an intramuscular electromyographic investigation into gluteus minimus and gluteus medius. Gait Posture. 2013; 38(1):157-60.
95. Semciw Al, Pizzari T, Murley GS, Green RA. Gluteus medius: an intramuscular EMG investigation of anterior, middle and posterior segments during gait. J Electromyogr Kinesiol. 2013;23(4):858-64.

96. Semciw A, Lawrenson P, Hoggarth MA, Hodges P, Vicenzino B, Crossley KM, King M, Heerey J, Elliott JM. Heterogeneous adiposity within gluteus minimus in healthy young adults. In: ASICS Sports Medicine Australia Conference: October 2017; Langkawi, Malaysia; 2017.

97. Semciw Al, Green RA, Pizzari T. Gluteal muscle function and size in swimmers. J Sci Med Sport. 2016;19(6):498-503.

98. Abbott R, Pedler A, Sterling M, Hides J, Murphey T, Hoggarth M, Elliott J. The geography of fatty infiltrates within the cervical multifidus and semispinalis cervicis in individuals with chronic whiplash-associated disorders. J Orthop Sports Phys Ther. 2015;45(4):281-8.

99. Smith AC, Parrish TB, Abbott R, Hoggarth MA, Mendoza K, Chen YF, Elliott JM. Muscle-fat MRI: 1.5 tesla and 3.0 tesla versus histology. Muscle Nerve. 2014;50(2):170-6.

100. Schulz KF, Altman DG, Moher D. CONSORT 2010 Statement: updated guidelines for reporting parallel group randomised trials. BMC Med. 2010;8(1):18.

101. Schulz KF, Grimes DA. Blinding in randomised trials: hiding who got what. Lancet. 2002;359(9307):696-700.

102. Ackerman IN, Kemp JL, Crossley KM, Culvenor AG, Hinman RS. Hip and knee osteoarthritis affects younger people, too. J Orthop Sports Phys Ther. 2017:47(2):67-79.

103. Perruccio AV, Power JD, Badley EM. Revisiting arthritis prevalence projections-it's more than just the aging of the population. J Rheumatol. 2006;33(9):1856-62.

\section{Ready to submit your research? Choose BMC and benefit from:}

- fast, convenient online submission

- thorough peer review by experienced researchers in your field

- rapid publication on acceptance

- support for research data, including large and complex data types

- gold Open Access which fosters wider collaboration and increased citations

- maximum visibility for your research: over $100 \mathrm{M}$ website views per year

At BMC, research is always in progress.

Learn more biomedcentral.com/submissions 